Swarthmore College

Works

1978

Rapidly Converging Activity Expansions For Representing The Thermodynamic Properties Of Fluid Systems: Gases, NonElectrolyte Solutions, Weak And Strong Electrolyte Solutions

R. H. Wood

T. H. Lilley

Peter T. Thompson

Swarthmore College, pthomps1@swarthmore.edu

Follow this and additional works at: https://works.swarthmore.edu/fac-chemistry

Part of the Chemistry Commons

Let us know how access to these works benefits you

Recommended Citation

R. H. Wood, T. H. Lilley, and Peter T. Thompson. (1978). "Rapidly Converging Activity Expansions For Representing The Thermodynamic Properties Of Fluid Systems: Gases, Non-Electrolyte Solutions, Weak And Strong Electrolyte Solutions". Journal Of The Chemical Society, Faraday Transactions 1: Physical Chemistry In Condensed Phases. 1301-1323. DOI: 10.1039/F19787401301

https://works.swarthmore.edu/fac-chemistry/186

This work is brought to you for free by Swarthmore College Libraries' Works. It has been accepted for inclusion in Chemistry \& Biochemistry Faculty Works by an authorized administrator of Works. For more information, please contact myworks@swarthmore.edu. 


\title{
Rapidly Converging Activity Expansions for Representing the Thermodynamic Properties of Fluid Systems: Gases, Non-electrolyte Solutions, Weak and Strong Electrolyte Solutions
}

\author{
BY ROBERT H. WOOD* \\ Department of Chemistry, University of Delaware, Newark, DE 19711, U.S.A. \\ AND \\ TERENCE H. LiLley \\ Department of Chemistry, The University, Sheffield S3 7HF \\ AND \\ PeTER T. THOMPSON \\ Department of Chemistry, Swarthmore College, Swarthmore, PA 19081, U.S.A.
}

Received 13th September, 1977

For dilute gases and non-electrolyte solutions in the McMillan-Mayer standard state, an activity expansion due to Mayer has great advantages over the normal concentration expansion (virial equation) for strongly associating species. For weakly interacting systems, both approaches are suitable. The activity expansion eliminates the need to differentiate between strong " chemical" interactions and weak "physical" interactions since the same equation is used in each situation.

The equation has been modified to represent electrolyte solutions in the McMillan-Mayer standard state by requiring that it be consistent with the Debye-Hückel and higher order limiting laws for strong electrolytes and that it be equivalent to a chemical association model for weak electrolytes. The result is a compact equation which contains no arbitrary ion-size parameters and which does not require the classification of an electrolyte as strong or weak. For 2:2 electrolytes, the equation gives a very good fit to the anomalous low concentration region.

For practical thermodynamic calculations, similar equations for molal activity coefficients are proposed; good fits of the data are obtained.

\section{INTRODUCTION}

An exact treatment of the interactions present in simple and complex fluid mixtures is required. In such systems, chemical equilibrium approaches are used when the interactions between species are strong, whereas " physical" non-ideality corrections are applied for weak interactions. ${ }^{1}$ Deciding which approach to use in a given situation is arbitrary. In equilibrium treatments for mixtures of electrolytes, this problem is particularly severe, and many predictions of the properties of these mixtures either treat the chemical equilibrium correctly, but approximate the nonideality corrections ${ }^{2}$ or do the reverse by considering only strong electrolyte mixtures. ${ }^{3}$ Because of these problems, it would be desirable to find an approach which treats both weakly and strongly interacting species without any formal changes in the equations. In this paper the utility of an approach involving an activity expansion rather than the more customary density (concentration) expansion is investigated for 
dilute gases and non-electrolyte solutions. The method is then modified for electrolyte solutions in such a way that it treats the limiting behaviour of both strong and weak electrolytes correctly. The usefulness of this formalism in representing the properties of electrolytes is then demonstrated. For strongly associating species, the activity expansion is much more rapidly convergent than the concentration expansion, in the sense that a smaller number of terms represents the data to higher concentrations. For weakly interacting species, neither expansion has any definite advantage.

Probably the most useful method of calculating the properties of electrolytes from the potential of average force involves the hypernetted chain equation. ${ }^{4}$ The calculations with this equation are at present too laborious for most practical purposes, particularly when mixtures of electrolytes are considered; furthermore, semiempirical potential functions are required. The present equations give compact and accurate expressions for representing the properties of complex mixtures but do not give detailed information about the potential of average force.

\section{DILUTE GASES AND NON-ELECTROLYTE SOLUTIONS}

The derivation of the concentration (number density) expansion for the thermodynamic properties of real gases starts with the Grand Canonical Ensemble, and has been outlined for the simple case of a one component imperfect gas by Hill. ${ }^{5}$ In this instance, the method is relatively simple and is worth reiterating at this point since to do so will clarify what comes later.

The Grand Canonical Partition Function (GCPF) can be written in terms of the Canonical Partition Function (CPF) and the absolute activity $(\lambda)$ as

$$
\mathrm{GCPF}=1+\sum_{N>0}(\mathrm{CPF})_{N} \lambda^{N}
$$

with $N=$ the number of molecules in the system and $\lambda=\exp (\mu / k T)$. Eqn (1) is a power series in the absolute activity $(\lambda)$ and may be transformed into a power series in the activity $(z)$ to give

$$
\mathrm{GCPF}=1+\sum_{N>0} \frac{(\mathrm{CI})_{N}}{N !} z^{N}
$$

where $(\mathrm{CI})_{N}$ is the configurational integral for $N$ molecules and $z=(\mathrm{CI})_{1} \lambda / V$. We also have

$$
\mathrm{GCPF}=\exp (p V / k T)
$$

consequently combining eqn (2) and (3) gives an expression for the pressure of a gas in terms of an activity expansion.

$$
p / k T=z+\sum_{n>1} b_{n} z^{n}
$$

The coefficients $b_{n}$ in this equation are well defined cluster integrals which may in principle be calculated exactly whenever the force law between particles is known.

Using the thermodynamic relation

$$
\rho=\left(\frac{\partial(p / k T)}{\partial \ln z}\right)_{T}
$$

where $\rho$ is the particle number density, we find

$$
\rho=z+\sum_{n>1} n b_{n} z^{n}
$$

Since $z$ is generally not known from experiments, the usual procedure at this point 
is to express this activity expansion as a concentration expansion by a series reversion using eqn (4) and (5). The new expression obtained is

$$
p / k T=\rho+\sum_{n>1} B_{n} \rho^{n}
$$

where the $B_{n}$ are the virial coefficients. Virial expansions like eqn (6) or modifications of it are widely used to represent thermodynamic data. The object of the present work is to indicate that the step from eqn (4) and (5) (the activity expansion) to eqn (6) (the concentration expansion) is not necessary and that eqn (4) and (5) have several advantages.

The general approach which has been used will first be outlined for gases and solutions containing non-electrolytes and examples of the utility of the approach will be given. The examples we have chosen are such that they illustrate particular points, but we have not intended that they should be comprehensive. We can treat both gases and non-ionic solutions using the same formalism since McMillan and Mayer ${ }^{6}$ have shown that the same expressions apply to both if, for the pressure of a gas, we substitute the osmotic pressure of the solution and if the solvent-averaged potential between isolated solute molecules is substituted for the direct potential. For a system containing the set $\boldsymbol{n}$ of molecular components, the pressure may be written as 7,8

$$
p / k T=\sum_{i} z_{i}+\sum_{n>1} b_{n} z^{n}
$$

Before explaining the nomenclature in this equation, it will be converted to molar units by defining

$$
K_{n} \equiv b_{n} N^{n-1}
$$

and a new activity, $a=z / N$ where $N$ is Avogadro's constant. Thus,

$$
p / R T=\sum_{i} a_{i}+\sum_{n>1} K_{n} a^{n} .
$$

The sum over $i$ is taken over all components and the sum over $\boldsymbol{n}$ is taken over all possible clusters of two or more molecules. A general cluster consists of $n_{1}$ molecules of component $1, n_{2}$ molecules of component 2 , etc. The symbol $a^{n}$ is defined as $a_{1}^{n_{1}} a_{2}^{n_{2}} a_{3}^{n_{3}} \ldots$... For example, in a two component system the clusters of 2 molecules would contribute $K_{11} a_{1}^{2}+K_{12} a_{1} a_{2}+K_{22} a_{2}^{2}$ to the sum. When the system is ideal, then $K_{n}=0$ for $n>1$ and

$$
p^{\mathrm{id}} / \boldsymbol{R} T=\sum_{i} c_{i}
$$

where $c_{i}$ is the molar stoichiometric concentration of the $i$ th species. If we now define the activity coefficient of a component $i$ by

$$
\gamma_{i}=a_{i} / c_{i}
$$

then

$$
p / R T=\sum_{i} c_{i} \gamma_{i}+\sum_{n>1} K_{n} c^{n} \gamma^{n}
$$

The first term on the right side of eqn (11) represents the contribution of the "free" components while the second term refers to the effect of " dimers", " trimers", etc., formed by molecular interaction. Similarly, for a multicomponent system, eqn (5) becomes

$$
c_{i}=a_{i}+\frac{1}{n>1} n_{i} K_{n} a^{n}
$$


where now $n_{i}$ denotes the number of $i$ molecules present in the various clusters formed from the set $n$. Thus, the activity coefficient of species $i$ is from eqn (10) and (12)

$$
\gamma_{i}=1-\left(\sum_{n>1} n_{i} K_{n} a^{n}\right) / c_{i} \text {. }
$$

If we now combine eqn (9) and (11), we get

$$
p / p^{\mathrm{id}}=\phi=\left[\sum_{i} c_{i} \gamma_{i}+\sum_{n>1} K_{n} c^{n} \gamma^{n}\right] / \sum_{i} c_{i}
$$

with $\gamma_{i}$ given by eqn (13). Eqn (14) is an expression for the compressibility coefficient in a gaseous system or the McMillan-Mayer osmotic coefficient of a non-electrolyte solution.

From eqn (11), it is readily shown by standard thermodynamic manipulations that the expression for the excess Helmholtz free energy $\left(A^{\text {ex }}\right)$ is

$$
A^{\mathrm{ex}} /(V R T)=\sum_{i} c_{i} \ln \gamma_{i}+\sum_{i} c_{i}\left(1-\gamma_{i}\right)-\sum_{n>1} K_{n} a^{n}
$$

Other thermodynamic quantities are obtainable from this, e.g., the excess internal energy, $U^{\text {ex }}$,

$$
U^{\mathrm{ex}} /(V \boldsymbol{R})=-\sum_{n>1} \frac{\mathrm{d} K_{n}}{\mathrm{~d}(1 / T)} a^{n} .
$$

The expressions obtained are complicated but take fairly simple forms if systems containing only one component (or one solute) are considered. Under this condition we obtain as the one component examples of eqn (8) and (12)-(14), respectively,

$$
\begin{aligned}
p / R T & =a+\sum_{n>1} K_{n} a^{n} \\
& =a+K_{2} a^{2}+K_{3} a^{3}+\ldots \\
c & =a+\sum_{n>1} n K_{n} a^{n} \\
& =a+2 K_{2} a^{2}+3 K_{3} a^{3}+\ldots \\
\gamma & =1-\left(\sum_{n>1} n K_{n} a^{n}\right) / c \\
& =1-2 K_{2} c \gamma^{2}-3 K_{3} c^{2} \gamma^{3} \ldots \\
p / p^{\text {id }} & =\phi=\gamma+\left(\sum_{n>1} K_{n} a^{n}\right) / c \\
& =\gamma+K_{2} c \gamma^{2}+K_{3} c^{2} \gamma^{3}+\ldots
\end{aligned}
$$

It is worthwhile at this point to compare the above expressions with those obtained using a concentration expansion. In particular, the analogues of eqn ( $\left.8^{\prime}\right)$ and $\left(14^{\prime}\right)$ are:

$$
\begin{aligned}
p / R T & =c+\sum_{n>1} B_{n} c^{n} \\
& =c+B_{2} c^{2}+B_{3} c^{3}+\ldots \\
\phi & =1+\sum_{n>1} B_{n} c^{n-1} \\
& =1+B_{2} c+B_{3} c^{2}+\ldots
\end{aligned}
$$

It should be noted that precise expressions linking any of the $B_{n}$ coefficients to the $K_{n}$ coefficients can be obtained. This will be referred to later.

So far we have equations for representing the properties of mixtures but no interpretation of the meaning of the various terms. Mayer ${ }^{8}$ (Section 4.5) has given such an interpretation by showing that the excess of associated pairs over those that 
would be expected for a random distribution is given by $\bar{N}_{2}=V N K_{2} a^{2}$. In general for any cluster of $n$ molecules, the excess of associated $n$-mers over that expected for a random distribution is given by $\bar{N}_{n}=V N K_{n} a^{n}$. Thus, eqn ( $\left.8^{\prime}\right)$ becomes

$$
p / k T=\sum_{n>0} \bar{N}_{n} / V
$$

in which $\bar{N}_{n} / V$ is the excess concentration for a cluster of $n$ molecules. Eqn (19) states that both the ideal gas law and the Van't Hoff law are always obeyed provided excess concentrations are used. Guggenheim has pointed out the usefulness of this equation and proposed the name "degree of sociation" for the excess association (positive or negative) above that which would be found in a random mixture. ${ }^{9}$ Similarly, he names the cluster integrals, $b_{n}$, the sociation constants. We shall follow Guggenheim in referring to $b_{n}$ or $K_{n}$ as sociation constants. As Guggenheim points out, these constants have the advantage that they are always well defined even when there is no strong association. In the limit of strong association, the normal association constant and the sociation constant are identical for all practical purposes while, for weakly interacting species, the association constant cannot be defined uniquely, whereas the sociation constant is always well defined. The fact that the sociation constant sometimes is negative has a simple physical interpretation; it just means that the excess (above random) concentration is negative. Whenever the action of the repulsive potential between molecules at very close distances produces a smaller concentration than would be expected from the bulk value, then the excess concentration in this region is negative. Unless this region is overwhelmed by the excess positive concentration at larger distances resulting from the positive part of the potential, the net excess concentration will be negative. As Guggenheim points out, ${ }^{9}$ the formula for a pair association constant, $K_{\mathrm{a}}$, is

$$
K_{\mathrm{a}}=\frac{N}{2} \int_{0}^{?}\langle\exp (-w / k T)\rangle 4 \pi r^{2} \mathrm{~d} r
$$

whereas the sociation constant, $K_{2}$, is

$$
K_{2}=\frac{N}{2} \int_{0}^{\infty}\langle\exp (-w / k T)-1\rangle 4 \pi r^{2} \mathrm{~d} r
$$

In the case of strong association, the arbitrary upper limit to the integration in eqn $\left(19^{\prime}\right)$ is not important as long as a reasonable value is used. However, for weak association, the association constant can be arbitrarily set by the choice of the integration limit. In the case of eqn $\left(19^{\prime \prime}\right)$, however, as the integral converges if $w(r)$ is not too long range, the sociation constant, $K_{2}$, is well defined and retains its rigorous interpretation as a measure of excess pair concentration.

The decision as to when to call a given configuration associated or not is quite arbitrary. Hill ${ }^{10}$ has shown that any arbitrary classification can be used and, provided that the statistical mechanics is done correctly, the overall thermodynamic properties of the mixtures are independent of the arbitrary separation. Hill has also shown that Mayer's definition of a cluster is just the definition that is necessary so that the clusters behave as if they were ideal; that is, this is the only definition for which the pressure of a non-ideal gas can be calculated from the sum of the concentrations of sociated species [eqn (19)].

The application of the above approach to single component gases and solutions is straightforward. Given a set of sociation constants, $K_{n}$, and a concentration, $c$, eqn $\left(13^{\prime}\right)$ is solved for the activity coefficients by standard non-linear techniques. Then the pressure is calculated from eqn (14). The approach has, in the present 
examples, been limited to situations where only the first two sociation constants are required adequately to describe a given set of experimental data, and this has introduced a subjective element insofar as the choice of concentration ranges is concerned. The inclusion of higher terms is relatively easy, but is not necessary for the present purposes. Thus, we have terminated data sets when the inclusion of measurements at higher concentrations significantly perturbed the reliability of the coefficients obtained from the least squares fitting procedure when only two sociation constants were used.

To obtain a better visual comparison of the relative efficacies of the activity and concentration expansions, it is useful to recast eqn $\left(14^{\prime}\right)$ and (18) into the forms

and

$$
(\phi-\gamma) / c \gamma^{2}=K_{2}+K_{3} a
$$

$$
(1-\phi) / c=-B_{2}-B_{3} c-B_{4} c^{2} \ldots
$$

where we have truncated the activity expansion but not the concentration expansion.

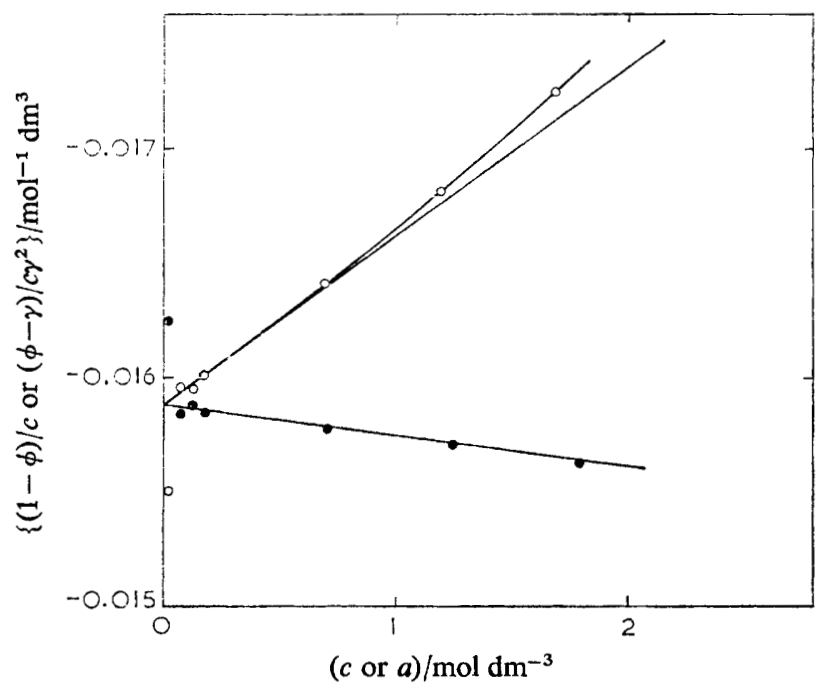

FrG. 1.-Plot of eqn (20) and (21) for argon at $700 \mathrm{~K}$. The intercept is $K_{2}=-B_{2}=-1.588$ $( \pm 0.002) \times 10^{-2}$ and corresponds to a negative sociation constant at this high temperature. $K_{3}=$ $1.346( \pm 0.060) \times 10^{-4}$. Experimental points are shown as closed (activity) and open (molarity) circles.

We first consider the properties of argon gas at two temperatures, 700 and $200 \mathrm{~K}$. The data ${ }^{11}$ were analysed using eqn $\left(14^{\prime}\right)$ and $(18)$ and these results, plotted in the forms of eqn (20) and (21), are given in fig. 1 and 2 . The intercepts in these figures are $K_{2}$ (or $-B_{2}$ ), and the slopes of the lines are $K_{3}$ (or $-B_{3}$ ). If higher terms are needed, the lines will not be straight at high concentrations. Also, since $K_{2}=-B_{2}$, the intercepts of the two plots must be identical. As can be seen from the figures, both the activity and concentration expansions adequately represent the data, although at $700 \mathrm{~K}$ the need for a triplet term is less pronounced in the activity expansion. This is as it should be since, for argon above its critical point, we would not expect long-lived dimers (or higher aggregates) to be of importance. Thus, each expansion works about equally well. At the higher temperature, the sociation constants are negative, corresponding to a net repulsive interaction between atoms, whereas, at the lower temperature, the converse applies. 
A similar analysis of the compressibility data ${ }^{12}$ of methane at $350^{\circ} \mathrm{C}$ is illustrated in fig. 3. Here again, the triplet term is smaller for the activity expansion and the requirement that both expressions have the same intercept suggests that terms higher than triplets are needed for the concentration expansion. However, the results do not suggest the formation of strong molecular clusters, which is consistent with our chemical knowledge of methane at temperatures above the critical point.

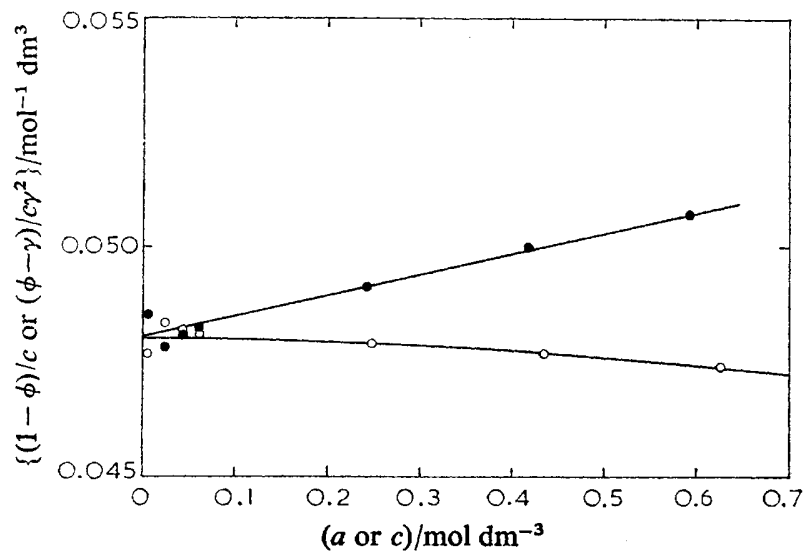

FIG. 2.-Plot of eqn (20) and (21) for argon at $200 \mathrm{~K}$. The intercept is $K_{2}=-B_{2}=4.806$ $( \pm 0.012) \times 10^{-2}$ and corresponds to a positive sociation constant at this low temperature. $K_{3}=$ $4.53( \pm 0.11) \times 10^{-4}$. Experimental points are shown as closed (activity) and open (molarity) circles.

We now turn to a rather more extreme example in the gas phase; namely, the interaction of nitrogen dioxide molecules. Unlike the earlier examples, the interaction between $\mathrm{NO}_{2}$ molecules is sufficiently intense, at low temperatures at least, that long-lived species $\left(\mathrm{N}_{2} \mathrm{O}_{4}\right)$ exist; indeed it has been customary to adopt a " chemical" approach to this system and treat the data ${ }^{13}$ obtained by an equilibrium constant treatment. The result was $K=79.4 \mathrm{dm}^{3} \mathrm{~mol}^{-1}$ at $35^{\circ} \mathrm{C}^{13}$ That such an

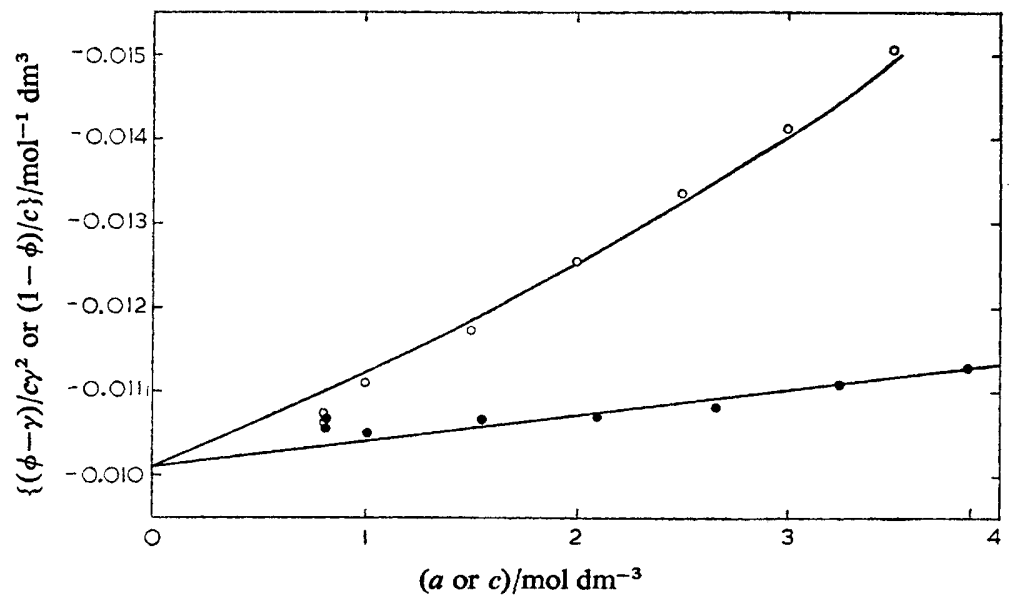

Fig. - 3.Plot of eqn (20) and (21) for methane at $350^{\circ} \mathrm{C}$. The intercept is $K_{2}=-B_{2}=-1.004$ $( \pm 0.028) \times 10^{-2}$ and corresponds to a negative sociation constant. $K_{3}=-3.14( \pm 0.45) \times 10^{-4}$. Experimental points are shown as closed (activity) and open (molarity) circles. 
approach is included in the present treatment is apparent from fig. 4 . Note that in this figure both sets of data must extrapolate to the same intercept $\left(K_{2}=-B_{2} \approx\right.$ 79.4). Two activity scale sociation constants are probably required to represent the experimental data, although a quite respectable fit was found using only the pair-wise interaction constant.

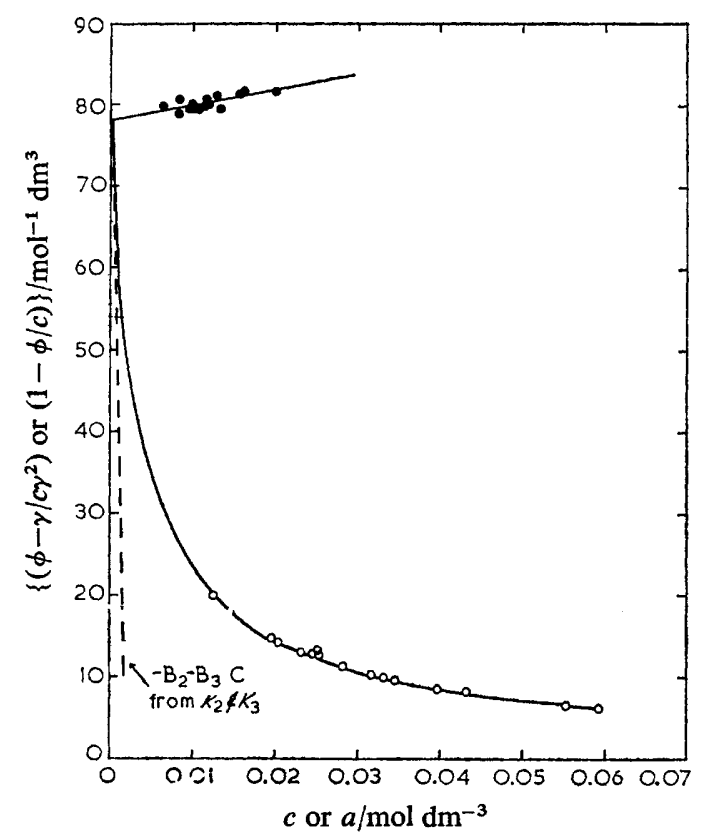

Fig. 4.-Plot of eqn (20) and (21) for $\mathrm{NO}_{2}$ at $35^{\circ} \mathrm{C}$. Experimental points are shown as closed (activity) and open (molarity) circles. Both solid lines are calculated using $K_{2}=78.06( \pm 5.78$ ) and $K_{3}=190( \pm 132)$ from an activity expansion [eqn (12)-(14)]. The dashed line represents eqn (21), using $B_{2}$ and $B_{3}$ only obtained from eqn (21) and the $K_{2}$ and $K_{3}$ values above. Note the excellent fit of the molarity data using just two constants $\left(K_{2}\right.$ and $\left.K_{3}\right)$ in an activity expansion.

This is in contradistinction to the concentration virial expansion which obviously requires many terms to obtain an adequate fit. As mentioned earlier, the sociation constants and concentration virial coefficients can be related by a series reversion $7,8,10$ and, if only the first two sociation constants are used, the expressions for the first few virial coefficients are :

$$
\begin{aligned}
& B_{2}=-K_{2} \\
& B_{3}=4 K_{2}^{2}-2 K_{3} \\
& B_{4}=-20 K_{2}^{3}+18 K_{2} K_{3} \\
& B_{5}=112 K_{2}^{4}-144 K_{2}^{2} K_{3}+18 K_{2}^{2} \\
& B_{6}=-672 K_{2}^{5}+952 K_{2}^{3} K_{3}-315 K_{2} K_{3}^{2} .
\end{aligned}
$$

The last three members of this set are incomplete when the $K_{n}$ for $n>3$ are no longer negligible. The results should be accurate in the present instance, however, because of the reasonable assumption that $K_{4}$ to $K_{6} \approx 0$ for $\mathrm{NO}_{2}$. Although the terms $B_{2}$ to $B_{6}$ would presumably give a reasonable approximation at the lowest concentrations if the data existed, at the actual experimental concentrations 
they are quite insufficient to represent the data. This arises because, when $K_{2}$ and $K_{3}$ are relatively large, the $B$ terms become very large and adjacent members of the series have alternating signs. In the present case, $B_{2}$ to $B_{6}$ are $-78.06,2.40 \times 10^{4}$, $-9.25 \times 10^{6}, 4.12 \times 10^{9}$ and $-2.12 \times 10^{12}$, respectively. It would seem that literally hundreds of terms might be required to represent the actual experimental concentration data adequately. However, if eqn (12)-(14) are rearranged to obtain $(1-\phi) / c$ and $c$, then just two activity expansion constants $\left(K_{2}\right.$ and $\left.K_{3}\right)$ are sufficient to calculate a curve which gives an excellent fit to the molarity data (see fig. 4). It should be noted that the numerical value obtained for the pairwise sociation constant is essentially the same as that obtained from an association treatment. ${ }^{13}$ This is as it should be ${ }^{9}$ for a strong localized pairwise interaction.

Our principal reason for pursuing the present approach stems from our interest in aqueous solutions containing non-electrolytes and/or electrolytes. As a first step in this regard, we have considered the properties of aqueous urea solutions at $25^{\circ} \mathrm{C}$, for which reliable and extensive thermodynamic data exist. ${ }^{14,15}$ When solutions are considered, a consistent treatment requires that the osmotic coefficient and the concentrations are those pertaining to McMillan-Mayer (MM) conditions. ${ }^{6}$ To stress this we adopt and extend the terminology used by Friedman ${ }^{16}$ to rewrite eqn (20) and (21) as

$$
\begin{gathered}
\left(\phi_{\mathrm{MM}}-\gamma_{\mathrm{MM}}\right) /\left(c_{\mathrm{MM}} \gamma_{\mathrm{MM}}^{2}\right)=K_{2, \mathrm{MM}}+K_{3, \mathrm{MM}} a_{\mathrm{MM}} \\
\left(1-\phi_{\mathrm{MM}}\right) / c_{\mathrm{MM}}=-B_{2, \mathrm{MM}}-B_{3, \mathrm{MM}} c_{\mathrm{MM}}-B_{4, \mathrm{MM}} c_{\mathrm{MM}}^{2}-\ldots
\end{gathered}
$$

The conversion ${ }^{16}$ of the experimental osmotic coefficient data from the molal, one atmosphere [Lewis-Randall (LR) $]^{16}$ scale to the MM scale was accomplished by the use of volumetric ${ }^{15}$ and isothermal compressibility data. ${ }^{17}$ It is worth noting that

Table 1.-Lewis-Randall (LR) and McMillan-Mayer (MM) osmotic coefficients at $25^{\circ} \mathrm{C}$ FOR UREA SOLUTIONS IN WATER

$\begin{array}{cccc}\begin{array}{c}m \\ / \mathrm{mol} \mathrm{kg}^{-1}\end{array} & \phi_{L R^{a}} & \begin{array}{c}c_{M M}{ }^{\circ} \\ / \mathrm{mol} \mathrm{dm}^{-3}\end{array} & \phi_{M M} \\ 1 & 0.9624 & 0.9558 & 1.0047 \\ 2 & 0.9331 & 1.8349 & 1.0157 \\ 4 & 0.8904 & 3.3951 & 1.0488 \\ 6 & 0.8607 & 4.7357 & 1.0957 \\ 8 & 0.8388 & 5.8979 & 1.1441 \\ 10 & 0.8299 & 6.9148 & 1.1921\end{array}$

$a$ Smoothed data from ref. (15). $b$ The molar concentration is at a pressure equal to the osmotic pressure of the solution.

the differences in the osmotic coefficients on the two scales are by no means insignificant. This is illustrated in table 1 in which observed LR and the correspondingly transformed $\mathrm{MM}$ values are given at the same round molalities. ${ }^{15}$ A superficial consideration of these urea data, using a molality representation, would lead one to believe that fairly extensive association was occurring in the solution, whereas, if the MM data are considered, these indicate that a net repulsive interaction exists between urea molecules. This problem in the analysis of data has been discussed previously. ${ }^{15,18}$ The idea that no net attractive interaction occurs between urea molecules in water is supported by spectroscopic evidence. ${ }^{19}$ In fig. 5 we illustrate the results for urea obtained by fitting the smoothed data tabulated by Stokes ${ }^{15}$ to eqn (22) and (23). The line drawn for the concentration expansion is that calculated 
from the sociation constant analysis [eqn $\left(21^{\prime}\right)$, together with $K_{2}$ and $K_{3}$ ]. It is apparent, however, that the activity expansion gives a satisfactory representation of the experimental data with a smaller numerical value of the " triplet "term than that obtained from a concentration expansion, although the concentration expansion has a slightly longer linear range.

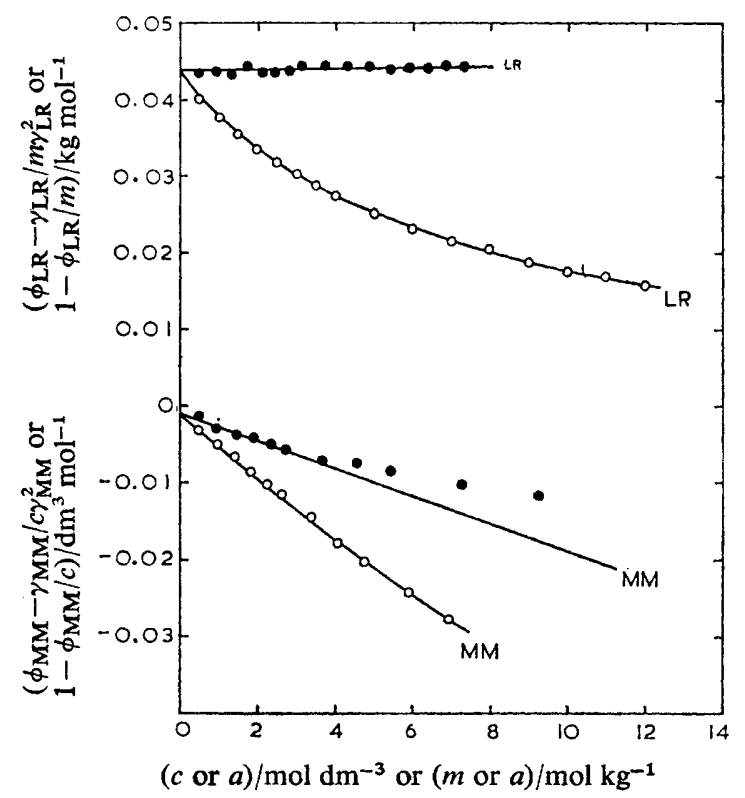

FIG. 5.-Lower lines are a plot of eqn (22) and (23) for aqueous urea in the McMillan-Mayer standard state. The concentration expansion in this case is more rapidly convergent. Upper figures are a plot of eqn (32) and (33) for aqueous urea in the Lewis and Randall standard state $(P=1 \mathrm{~atm})$ against molality. In this case, the activity expansion is much more rapidly convergent. Experimental points are shown as closed (activity) and open (molarity) circles.

The above examples show that the activity expansion is as rapidly convergent as the concentration expansion in most cases, and that for strongly associating species it is very much more rapidly convergent.* The reasons for this convergence can be explained from a variety of viewpoints, some of which we discuss below.

In the case of a species which associates very strongly to form dimers with negligible higher terms, the reason for the increased convergence of the activity expansion is evident by comparing the first terms in the two expansions. In the density expansion [eqn (17)], the concentration of dimer is approximated by the " equilibrium constant ", $B_{2}$, times the concentration of the monomer squared. This is correct only in dilute solutions when the concentration of the monomer is approximately equal to its activity. In the corresponding activity expansion [eqn (11)], the concentration of dimer is calculated as the equilibrium constant, $K_{2}$, times the activity of monomer squared. This is correct at all concentrations. As an extreme example of this kind of behaviour, consider a system of $I_{2}$ vapour viewed as being composed of $I$ atoms with strong pairwise association. It would be difficult to find a convergent density expansion, but the activity expansion would only need one term, the pairwise

* It is more rapidly convergent in the sense that fewer terms are needed in the series and that it represents data over a wider concentration range. 
association term, to represent the system up to moderate pressures. Another way of saying the same thing is to note that the pairwise association constant, $K_{2}$, appears in all of the higher virial coefficients of the concentration expansion [see eqn $\left(21^{\prime}\right)$ ]. Thus, if $K_{2}$ is very large and all of the other $K$ values are small, then the activity expansion converges very rapidly, whereas the density expansion converges very slowly. This is just the situation with $I_{2}$ considered as a monoatomic gas. Of course, in this extreme example a "chemist" would know that gaseous iodine is diatomic and hence know not to choose I atoms as the primary component of the system. But the use of this type of privileged knowledge is precisely what we are attempting to avoid in the present approach.

Looking at the convergence properties from the statistical mechanical point of view, the difference between the activity expansion and the density expansion is that they each represent different resummations of the graphs of the grand canonical ensemble expression. ${ }^{10}$ The activity expansion is much more rapidly convergent in the case of monoatomic iodine because the two iodine atoms tend to form one and only one strong bond. That is, when two iodine atoms approach, they form a chemical bond and another iodine atom approaching the resulting $\mathrm{I}_{2}$ molecule cannot bond to this molecule. This is an extreme case of a non-pairwise additive potential. Thus, the dimerization of iodine atoms is very strong compared to the formation of higher aggregates and, in this case, the resummation implied by the activity expansion is much more rapidly convergent that the density expansion.

Recently, Anderson ${ }^{20}$ in an investigation of the topological properties of cluster diagrams for hydrogen bonded fluids has shown that a chemical equilibrium model can be used as an appropriate guide to the way in which the cluster diagrams should be resummed and to the way in which certain diagrams cancel each other. Since the same argument applies to any cluster which can form as a limited number of "bonds", there is a direct connection between the way certain cluster diagrams cancel each other and the chemical association model. From this argument, it is concluded that the activity expansion with terms for pairs and triplets ( $K_{2}^{\circ}$ and $\left.K_{3}^{\circ}\right)^{*}$ will be rapidly convergent for any strongly associating system in which aggregates greater than triplets are not important.

The above discussion suggests that, using chemical association as a guideline, the activity expansion can be modified to fit many special situations. As one common example, consider a system which associates strongly and can form very large aggregates (for example, aqueous solutions of nucleic acids). The chemical association model is

$$
\begin{aligned}
& 2 \mathrm{~A} \stackrel{K_{2}^{\circ}}{\rightleftharpoons} \mathrm{A}_{2} \\
& \mathrm{~A}_{2}+\mathrm{A} \stackrel{K_{3}^{\circ}}{\rightleftharpoons} \mathrm{A}_{3} \\
& \mathrm{~A}_{n-1}+\mathrm{A} \stackrel{K_{n}^{\circ}}{\rightleftharpoons} A_{n} .
\end{aligned}
$$

If we make the usual assumption that the equilibrium constants are related by $K_{n}=K_{3} \neq K_{2}$, the data for many real systems can be fitted by this chemical equilibrium model. ${ }^{21,22}$ The corresponding activity expansion [eqn (12)] becomes

$$
\begin{aligned}
c & =a+2 K_{2}^{\circ} a^{2}+3 K_{2}^{\circ} K_{3}^{\circ} a^{3}+4 K_{2}^{\circ} K_{3}^{\circ 2} a_{4}^{4}+5 K_{2}^{\circ} K_{3}^{\circ 3} a^{5}+\ldots \\
& =a\left(1-\frac{K_{2}^{\circ}}{K_{3}^{\circ}}\right)+a \frac{K_{2}^{\circ}}{K_{3}^{\circ}}\left(1+2 K_{3}^{\circ} a+3 K_{3}^{\circ 2} a^{2}+4 K_{3}^{\circ} a^{3}+\ldots\right)
\end{aligned}
$$

* $K_{n}^{\circ}$ is a stepwise association constant, whereas $K_{n}$ is an overall association constant. 
and using $1 /(1-x)^{2}=\left(1+2 x+3 x^{2}+4 x^{3}+\ldots\right)$, we obtain

$$
c=a\left(1-\frac{K_{2}^{\circ}}{K_{3}^{\circ}}\right)+\frac{a K_{2}^{\circ}}{K_{3}^{\circ}\left(1-K_{3}^{\circ} a\right)^{2}} .
$$

This resummation of eqn (12) is just the one which should be useful when the chemical approximations used in deriving it are reasonable.

\section{ELECTROLYTE SOLUTIONS}

The previous discussion has shown that an activity expansion can represent the properties of systems with strongly associating species as well as the properties of systems where volume exclusion is the main contribution to the virial expansion. One of the continuing problems in the representation of the properties of electrolyte solutions is the question of at what precise point we call an electrolyte "weak" rather than "strong". The decision is always arbitrary, and when the shift is made it is necessary to introduce new parameters to take into account the change from "strong" to "weak". Consider, for example, the complexity of a system in which this shift occurs as a function of temperature. For this reason (as well as for the other advantages of the activity expansion) it was of interest to see if the same ideas could be applied to electrolyte solutions.

In order to do this, it is convenient to consider eqn(13) as the fundamental equation for an activity expansion.* For a non-electrolyte solution containing $\sigma$ solute components, this equation represents a series of $\sigma$ non-linear equations for the activity coefficients of the $\sigma$ species in terms of their concentrations and their sociation constants $\left(K_{n}\right)$. Once the activity coefficients are calculated by solving this set of non-linear equations, all of the other thermodynamic properties follow and can be calculated by eqn (14), (15), (16), etc. This is similar in procedure and degree of difficulty to the solution of a chemical equilibrium problem. In adapting eqn (13) for the representation of the properties of electrolyte solutions, the following constraints were used:

(1) In the limit of low concentration, the equation must approach the DebyeHückel limiting law.

(2) The equation must be compatible with the concentration dependence of the terms beyond the limiting law which have been derived rigorously from cluster expansion methods. ${ }^{24,25}$

(3) In the limiting case of very strong association (e.g., acetic acid), the equation must be equivalent to an ion-association model. ${ }^{2,3}$

The only reasonable form that we have been able to find for eqn (13), given these constraints is

$$
\gamma_{i} c_{i}=a_{i}=\gamma_{i}^{\circ}\left[c_{i}-\sum_{n>1} a^{n} n_{i} K_{n} / \gamma_{n}^{\circ}\right]
$$

where $\gamma_{i}^{\circ}$ and $\gamma_{n}^{\circ}$ are Debye-Hückel type terms which correct for the reduction in the activity of the species $i$ and the cluster $n$, respectively due to the ion atmosphere effects. $\dagger$ Considering the case where the sociation constants are positive, eqn (26) is most easily understood if the right-hand side is thought of an being the product of the "free" ion $i$ concentration times the "free" ion activity coefficient, $\gamma_{i}^{\circ}$. The former is the stoichiometric concentration, $c_{i}$, minus the concentration of $i$ present

\footnotetext{
* With this change, $\gamma$ becomes the fundamental quantity rather than $A^{\mathrm{ex}}$. There are some disadvantages in this change. ${ }^{23}$

$\dagger$ It should be noted that the $\gamma_{i}^{\bullet}$ and $\gamma_{n}^{\circ}$ are not the stoichiometric activity coefficients. They are corrections for ion atmosphere effects and are equal to 1 for uncharged species.
} 
in " dimers", "trimers", etc. According to the first constraint, $y_{i}^{\circ}$ must reduce to the Debye-Hückel limiting law value at low concentrations. One possible choice for $\gamma_{i}^{\circ}$ would be the limiting law itself, i.e., $\gamma_{i}^{\circ}=\exp \left(-A_{\gamma} Z_{i}^{2} I^{\frac{1}{2}}\right)$ with $I=\frac{1}{2} \sum_{i} c_{i} Z_{i}^{2}$.

However, this choice for ionic strength, $I$, is clearly not acceptable for strongly associating systems. Consider, for example, a 1 molar acetic acid solution which, on the present model, would contain as the components one molar stoichiometric concentration of $\mathrm{H}^{+}$and $\mathrm{Ac}^{-}$. The resulting concentration ionic strength $(I=1.0)$ would be too large and $\gamma_{i}^{\circ}$ too small; knowing what we do about the tendency of $\mathrm{H}^{+}$and $\mathrm{Ac}^{-}$to associate a better guess would be $I=0$ and $\gamma_{i}^{\circ}=1$. In the spirit of the present development, we propose to use the Debye-Hückel Limiting Law expression, but with concentrations in the ionic strength term replaced by ionic activities. The result is :

$$
\gamma_{j}^{\circ}=\exp \left(-A_{\gamma} Z_{j}^{2} \sqrt{I_{\mathrm{a}}}\right)
$$

and

$$
I_{\mathrm{a}}=\left(\frac{1}{2}\right)\left(\sum_{i} a_{i} Z_{i}^{2}+\sum_{n>1} a_{n} Z_{n}^{2}\right)
$$

with

$$
a_{n}=K_{n} a^{n}=K_{n} c^{n} \gamma^{n}
$$

where $A_{\gamma}$ is the Debye-Hückel limiting law slope, $Z_{j}$ is the charge on ion $j$, and $I_{\mathrm{a}}$ is the ionic strength expressed in terms of activities rather than concentrations. The first term on the right-hand side of eqn (28) is the effective ionic strength due to the original ionic components $(i)$, and the second sum is the effective ionic strength due to aggregates of ions (clusters) that have formed in the solution. It is easily shown (see Appendix) that, in the limit of very low concentrations, eqn (27) and (28) approach the Debye-Hückel limiting law. At slightly higher concentrations only the first term in eqn (28) contributes significantly and the result is then just the Debye-Hückel limiting law with $I_{\mathrm{a}}$ expressed in terms of the activities of the original ionic components. The limiting law in this case has been derived by Vedenov ${ }^{26}$ and also by Jones and Molling ${ }^{27,28}$ using a resummation of cluster integrals in an activity expansion. Thus, a Debye-Hückel expression using activities in the ionic strength term has just as much theoretical basis as one using concentration. As Jones and Molling ${ }^{27}$ have pointed out, in actual fact, the activity expression is much better at higher concentrations in representing the properties of real ionic solutions. This is not surprising because in a qualitative way eqn (28) assumes that the effectiveness of an ion in contributing to the formation of an atmosphere around another ion is proportional to its activity, not its concentration. The second term in eqn (28) has not been derived on any theoretical basis but, as will be shown below, it is consistent with the known form of the density expansion of electrolyte solutions (the first and second constraints), and it must be present in order to satisfy the third constraint. The latter point is easiest to see by picking an extreme case. Consider, for example, the properties of mercurous chloride thought of as consisting of ionic components $\mathrm{Hg}^{+}$and $\mathrm{Cl}^{-}$(certainly at low enough concentrations, this salt would be a 1-1 electrolyte). The apparent ionic strength in a 0.1 molal solution would be $0.1 \mathrm{~mol} \mathrm{dm}^{-3}$. However, we know that due to association the actual species present are $\mathrm{Hg}_{2}^{2+}\left(0.05 \mathrm{~mol} \mathrm{dm}^{-3}\right)$ and $\mathrm{Cl}^{-}\left(0.1 \mathrm{~mol} \mathrm{dm}^{-3}\right)$ so that the true ionic strength would be $0.15 \mathrm{~mol} \mathrm{dm}^{-3}$. With the use of eqn (26) and (27), the calculated contributions of the first and second terms in eqn (28) to $I_{\mathrm{a}}$ are about 0.05 and $0.10 \mathrm{~mol} \mathrm{dm}^{-3}$, respectively. Thus, we see that both terms in eqn (28) are necessary to obtain the 
right answer and that in the case of extreme association to a charged dimer $\left(\mathrm{Hg}_{2}^{2}{ }^{+}\right)$ the second term can contribute even more to $I_{\mathrm{a}}$ than does the first.

We now turn back to eqn (26) and show how the third constraint dictates the way in which the ion atmosphere terms are added to eqn (26). For simplicity, we consider the case of a weak electrolyte, aqueous acetic acid, which in terms of eqn (26) is a two solute component solution. The components are the principal species believed to be present at infinite dilution as revealed by conductance measurements; namely, $\mathrm{H}^{+}$and $\mathrm{Ac}^{-}$. Applying eqn (26) to the activity of hydrogen ion in such a solution, we have :

$$
a_{\mathrm{H}^{+}}=\gamma_{\mathrm{H}^{+}}^{\circ}\left\{c_{\mathrm{H}^{+}}-\left[a_{\mathrm{H}^{+}} a_{\mathrm{Ac}^{-}} K(\mathrm{HAc}) / \gamma_{\mathrm{HAc}^{\circ}}^{\circ}\right]-\left[a_{\mathrm{H}^{+}} a_{\mathrm{Ac}^{2}}^{2} K\left(\mathrm{HAc}_{2}^{-}\right) / \gamma_{\mathrm{HAc}_{2}^{-}}^{\circ}\right]\right\}
$$

where $K(\mathrm{HAc})$ is the association constant of acetic acid and, for simplicity, we have included only the pairwise association of HAc $[K(\mathrm{HAc})]$ and one triplet term, $K\left(\mathrm{HAc}_{2}^{-}\right)$. The ion-pairing model interpretation of eqn (29) is as follows : the term in curly brackets on the right side is the "free " $\mathrm{H}^{+}$concentration. It consists of the stoichiometric $\mathrm{H}^{+}$concentration, $c_{\mathrm{H}^{+}}$, minus the concentration of $\mathrm{H}^{+}$contained in the clusters $\mathrm{HAc}$ and $\mathrm{HAc}_{2}^{-}$. For example, since

$$
\begin{aligned}
K\left(\mathrm{HAc}_{2}^{-}\right) & =a_{\mathrm{HAc}^{-}} / a_{\mathrm{H}^{+}} a_{\mathrm{Ac}^{-}}^{2}, \\
a_{\mathrm{HAc}_{2}^{-}} / \gamma_{\mathrm{HAc}^{-}}^{\circ} & =c_{\mathrm{HAc}^{-}}=a_{\mathrm{H}^{+}} a_{\mathrm{Ac}^{2}}^{2} K\left(\mathrm{HAc}_{2}^{-}\right) / \gamma_{\mathrm{HAc}_{2}^{-}}^{\circ} .
\end{aligned}
$$

Dividing by the $\gamma^{\circ}$ converts an activity to a free concentration because in an ion association model the activity is just the free concentration times the Debye-Hückel activity coefficient. In the case of acetic acid, $\gamma_{\mathrm{HAc}}^{\circ}$ equals 1 since this is an uncharged species and there is no Debye-Hückel screening. However, such a term is necessary for the charged aggregate $\mathrm{H}(\mathrm{Ac})_{2}^{-}$, which is included in the third term on the right hand side of eqn (29). In the ion-association model, the $\mathrm{H}^{+}$activity is then given by the free concentration times the activity coefficient of the "free "ion, $\gamma_{\mathrm{H}^{+}}^{\circ}$. It should also be noted that it is just the presence of these $\gamma^{\circ}$ terms that produces Friedman's limiting law of charge-symmetric mixtures. ${ }^{29}$

Eqn (26), (27) and (28) are a compact set of equations for representing the properties of any mixture containing both electrolytes and non-electrolytes. The equations have the disadvantage that they are non-linear and, in the multi-component case, must be solved via a complex computer program. However, this is also true of any ion-association model for strongly associating solutions. It is also true for the more sophisticated equations using density expansions to represent the properties of mixtures of strong electrolytes. ${ }^{3}$ We believe that this disadvantage is far outweighted by the following advantages:

(1) the system of equations is a compact expression that represents the properties of any mixture of non-electrolytes, strong electrolytes, and weak electrolytes without any arbitrary assumption as to whether or not an electrolyte should be classified as weak or strong. This is particularly important when investigating a range of temperatures over which an electrolyte changes from strong to weak.

(2) No arbitrary ion size parameters appear in our equations so that no ad hoc assumptions about their values are necessary.

(3) Compared with the usual density expansions for the activities of 2:2 electrolytes, the present equations can represent their thermodynamic properties up to much higher concentrations with fewer parameters.

(4) Using the concepts in these equations, the authors have found that it is much easier to think about the properties of complex mixtures, since there are no arbitrary divisions into non-electrolytes, strong electrolytes and weak electrolytes. The 
following examples illustrate the utility of these equations for representing the properties of electrolyte solutions.

We begin by noting that one of the most striking deficiencies of the usual density expansions is that they do not give good fits of data for $2: 2$ electrolytes at low concentrations. The difficulty is illustrated in fig. 6 where the cell data of Rasaiah for $\mathrm{ZnSO}_{4}{ }^{30}$ are plotted in such a way that they must approach the Debye-Hückel limiting slope at $m=0.0^{30,31}$ At low concentrations, the experimental points show an apparent slope greater than the Debye-Hückel slope, but this quickly shifts to apparent slopes less than the Debye-Hückel slope at concentrations above $0.007 \mathrm{~mol} \mathrm{dm}^{-3} .^{31}$ Attempts to fit this data to a density expansion including the Debye-Hückel limiting slope have failed. ${ }^{30}$ Because of this difficulty, Rasaiah used a cube-root law to fit the low concentration data together with an assumed smooth transition to the Debye-Hückel limiting law. The cube-root law was used because it gives a very good fit to the low concentration data of a number of salts which show the same kind of deviations from the Debye-Hückel behaviour. In the case of Rasaiah's zinc sulphate data, the cube root law fits the first five experimental points using two adjustable constants with a standard deviation of $73 \mu \mathrm{V}$. Assuming a smooth transition to the Debye-Hückel limiting law then gives an extrapolated $E^{\circ}$ for the cell of $412.31 \mathrm{mV}$. The present equations* fit the same data with essentially the same accuracy and the same number of adjustable constants over a much wider concentration range. For instance, using the $E^{\circ}$ of the cell together with one sociation constant for the cation and anion $\left[K\left(\mathrm{ZnSO}_{4}\right)\right]$, the first five data points can be fitted with a standard deviation of $82 \mu \mathrm{V}$. As more experimental points are added, the standard deviation of the fit and the $95 \%$ confidence limits of $E^{\circ}$ and $K\left(\mathrm{ZnSO}_{4}\right)$ decrease until 8 points are reached and then increase. Our conclusion is that the fit with 8 points is the best two constant extrapolation of the data. The resulting $E^{\circ}$ is $412.55 \pm 0.40 \mathrm{mV}$ and the standard deviation of the fit is $85 \mu \mathrm{V}$. This is essentially as good a fit as the cube root law which covers only the first five points (maximum concentration $0.0039 \mathrm{~mol} \mathrm{dm}^{-3}$ as compared with $0.0088 \mathrm{~mol} \mathrm{dm}^{-3}$ ). The present equations, therefore, give a good fit of the low concentration data for zinc sulphate with only a pairwise sociation constant. Fig. 6 shows a plot of the fit to the first 8 points and the way in which the present equations approach the Debye-Hückel limiting slope. It should be noted that there are no systematic deviations of the experimental points from the least squares fit. The problem with fitting these data with a density expansion is evident from fig. 6 which shows that the data points cross the Debye-Hückel limiting law (DHLL) at about $0.007 \mathrm{~mol} \mathrm{~kg}^{-1}$. Thus, the term linear in $m$ is called upon to perform the impossible task of representing both positive and negative deviations from the limiting law. However, the activity limiting law (ACTLL) [eqn (26)-(28) with all $K_{n}=0$ ] shown in fig. 6 is always below the experimental points at low concentrations, so that a single sociation constant can easily fit the data to $0.009 \mathrm{~mol} \mathrm{dm}^{-3}$. There are a few 1-1 electrolytes which also show negative deviations from the Debye-Hückel limiting slope at very low concentrations; it is probable that the present equations can also fit these data.

* Eqn (26)-(28) were adopted to fit the cell data of Rasaiah by first rearranging the cell equation to $E^{\prime}=E^{\circ}-k^{\prime} \ln \gamma_{ \pm}^{\mathrm{LR}}$, where $E^{\prime}$ is an experimentally known function of the observed e.m.f. and the concentrations of $\mathrm{Zn}^{2+}$ and $\mathrm{SO}_{4}^{2-}$ and $k^{\prime}=R T / F$. Using the equations of Friedman ${ }^{16}$ for the corrections from the Lewis-Randall (LR) standard state to the McMillan-Mayer (MM) standard state results in $E^{\prime \prime}=E^{\circ}-k^{\prime} \ln \gamma_{ \pm}^{M M}$ where $E^{\prime \prime}$ is again an experimentally known quantity. The least-squares fitting method involves finding the best values of $E^{\circ}$ and the $K$ values of eqn (26)-(28) needed to minimize the deviations in $E^{\prime \prime}$. This procedure gives $E^{\circ}$ on the molality scale at one atmosphere pressure because the fit is the same as that obtained by correcting the activity coefficients on the MM scale to the LR scale and then fitting the data. 
A similar procedure can be used with three constants: $E^{\circ}, K\left(\mathrm{ZnSO}_{4}\right)$ and $K\left(\mathrm{Zn}_{2} \mathrm{SO}_{4}^{2+}\right) .^{*}$ Again, points were added to the fit until the $95 \%$ confidence limits of $E^{\circ}$ and the $K$ values reached a minimum. Eliminating one experimental point at $0.00887 \mathrm{~mol} \mathrm{~kg} \mathrm{k}^{-1}$ (which seems to be in error by over $2 \mathrm{mV}$ ) the best three constant fit occurs with 14 data points to $m(\max )=0.24 \mathrm{~mol} \mathrm{dm}{ }^{-3}$. The resulting $E^{\circ}$ is $412.35 \pm 0.50 \mathrm{mV}$, and the standard deviation of the fit is $130 \mu \mathrm{V}$. Because of its lower $95 \%$ confidence limits, we believe the two constant extrapolations to be slightly superior. However, both extrapolations together with the cube root extrapolation of Rasaiah yield the same results well within the $95 \%$ confidence limits. The values for $K\left(\mathrm{ZnSO}_{4}\right)$ derived by both activity expansion extrapolations are also very close together: $153 \pm 18$ and $161 \pm 21 \mathrm{~kg} \mathrm{~mol}^{-1}$. These sociation constants are also consistent with a variety of measurements of the association constant for $\mathrm{ZnSO}_{4}$.

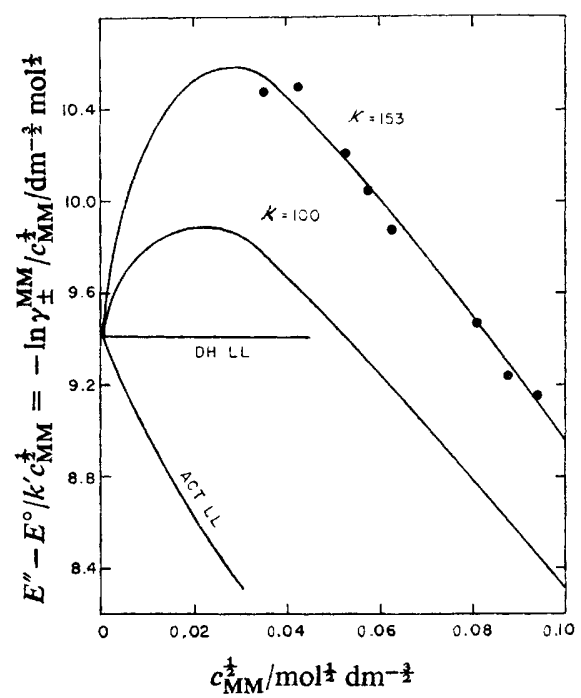

Fig. 6.-Plot of $\left(E^{\prime \prime}-E^{\circ}\right) / k^{\prime} c^{\frac{1}{2}}=-\ln \left(\gamma_{ \pm}\right) / c^{\frac{1}{2}}$ for the $\mathrm{ZnSO}_{4}$ cell data of Rasaiah. The intercept is the Debye-Hückel limiting slope. The Debye-Hückel limiting law is denoted by DHLL, and the activity limiting law by ACTLL. (This limiting law is given by eqn (26)-(28) with all $K$ values $=$ $0)$. Solid lines also give the values of $-\ln \left(\gamma_{ \pm}\right) / c^{\frac{1}{2}}$ from eqn $(26)-(28)$ with $K\left(\mathrm{ZnSO}_{4}\right)=100$ and with $K\left(\mathrm{ZnSO}_{4}\right)=153$. Note the apparently random deviations of the experimental points from the curve with $K\left(\mathrm{ZnSO}_{4}\right)=153$.

Brown and Prue ${ }^{32}$ surveyed previous literature and found values ranging from 222 to $154 \mathrm{~kg} \mathrm{~mol}^{-1}$ using e.m.f., solubility, conductance and optical techniques. Brown and Prue also found values from 100 to $238 \mathrm{~kg} \mathrm{~mol}^{-1}$, depending on what assumptions were made about the distance of closest approach to be used in the Debye-Hückel equation. The results of these fits indicate that the activity coefficient of zinc sulphate can be fitted adequately with a pairwise sociation constant up to $0.009 \mathrm{~mol} \mathrm{dm}^{-3}$ and that adding a triplet sociation constant increases the range which can be fit up to $0.24 \mathrm{~mol} \mathrm{dm}^{-3}$. It seems likely that a chemical equilibrium model would also be able to extrapolate the cell data of Rasaiah since the problem with the density expansion is the strong association of these salts. Lilley and Briggs ${ }^{33}$ have shown that this approach works well in the case of calcium sulphate. The advantage of

* This is the sociation constant for $2 \mathrm{Zn}^{2+}+\mathrm{SO}_{4}^{2-} \rightarrow \mathrm{Zn}_{2} \mathrm{SO}_{4}^{2+}$. The equations cannot distinguish between this and $K\left[\mathrm{Zn}\left(\mathrm{SO}_{4}\right)_{2}^{2-}\right.$. 
the present approach is that it is not necessary to decide ahead of time whether the salt is strongly associated, and that if it is associated it is not necessary to use any arbitrary ion size parameters for the " unassociated" electrolyte.

As an example of the ability of the equations to fit data for $1: 1$ electrolytes, the cell data of Gupta, Hills and Ives ${ }^{34}$ on $\mathrm{HCl}$ solutions were fitted in a similar manner after converting to the McMillan-Mayer state. The best extrapolation with two adjustable parameters was for 4 experimental points $\left(m_{(\max )}=0.028 \mathrm{~mol} \mathrm{~kg}^{-1}\right)$ giving $E^{\circ}=268.27 \pm 0.06 \mathrm{mV}$ and $K\left(\mathrm{H}^{+}, \mathrm{Cl}^{-}\right)=-1.544 \pm 0.09$ with a standard deviation of the fit of $0.01 \mathrm{mV}$. The best extrapolations with three adjustable parameters was with 10 experimental points $\left(m_{(\max )}=0.098 \mathrm{~mol} \mathrm{~kg}^{-1}\right)$. Using two different pairwise sociation constants, the results were $E^{\circ}=268.29 \pm 0.15 \mathrm{mV}, K\left(\mathrm{H}^{+}, \mathrm{Cl}^{-}\right)=-1.415 \pm$ 0.31 and $K\left(\mathrm{H}^{+}, \mathrm{H}^{+}\right)=-0.0964 \pm 0.06$ with a standard deviation of fit of $0.049 \mathrm{mV}$. Using one triplet sociation constant gave $E^{\circ}=268.30 \pm 0.15 \mathrm{mV}, K\left(\mathrm{H}^{+}, \mathrm{Cl}^{-}\right)=$ $-1.589 \pm 0.20$, and $K\left(\mathrm{H}^{+}, \mathrm{Cl}^{-}, \mathrm{H}^{+}\right)=-1.404 \pm 1.02$ with a standard deviation of $0.05 \mathrm{mV}$. These results show that the activity expansion is about as good as the density expansion. Using a density expansion, Gupta, Hills and Ives ${ }^{34}$ found $E^{\circ}=268.18 \mathrm{mV}$. These results show that for unassociated electrolytes the activity expansion is not superior to the usual density expansion with an ion-size parameter. It seems probable that the large volume exclusion effects in strong electrolytes ( $K_{+-}$is negative) require quartet terms above about $m=0.10 \mathrm{~mol} \mathrm{~kg}^{-1}$.

There are a number of possible ways of extending the utility of the present equations for electrolyte solutions which we have not yet had the time to explore. Two of these are briefly discussed below.

(1) For charged asymmetric mixtures there is a known higher order limiting law 24, 25 which gives a term proportional to $c \ln c$ for the logarithm of the activity coefficient. This limiting law is a function only of the concentration, the ions and the properties of the solvent. For this reason it seems quite likely that it will be useful, in the case of charge-asymmetric mixtures to include this limiting law in eqn (27) (again replacing concentrations by activities). This would give a screening term that includes all of the known effects that are only dependent on the properties of the solvent, as required by constraint 2 . Adding this term to the equations would build in to them all of the higher order limiting laws for mixtures discussed by Friedman. ${ }^{24,} 25,35$

(2) Because of the way in which eqn (26) treats the ions as components, there is the possibility of getting a rational set of single ion activity coefficients from this equation. These would be based on the convention that the single-ion activity coefficient approaches 1 as the concentration goes to zero. Using the hypernetted chain equation in the appropriate form for electrolyte solutions, Friedman ${ }^{\mathbf{3 6}}$ has shown that it is possible, at least roughly, to separate out the effects of oppositely charged ion interactions from like-charged ion interactions. However, it was not possible with the data available to separate out which effects were due to interactions between two positive charges or two negative charges. For a symmetrical electrolyte, eqn (26) has the same properties but, for charge-asymmetric electrolytes such as $\mathrm{MgCl}_{2}$, the three pairwise interactions in eqn (26) have different concentration dependences. The difference in concentration dependence is in the $K_{n} / \gamma_{n}^{\circ}$ term: the $\mathrm{MgCl}^{+}$pair has a charge of +1 , the $\mathrm{MgMg}^{4+}$ pair has a charge of +4 , and the $\mathrm{ClCl}^{2-}$ pair has a charge of -2 . Thus, the Debye-Hückel screening effects $\left(\gamma^{\circ}\right)$ for these three pairs have differing concentration dependences and, at least in principle, they can be separated if the activity coefficients are known with sufficient accuracy. This possibility is also implicit in earlier attempts to add $\gamma^{\circ}$ to an association constant model. ${ }^{37}$ 


\section{EQUATIONS APPLICABLE TO CONDITIONS OF CONSTANT PRESSURE}

For most practical calculations of equilibria in complex natural mixtures, the present equations are awkward because of the need for corrections to the McMillanMayer standard state. The purpose of this section is to point out that the equations with the concentration, $c$, replaced by the molality, $m$, have just as much theoretical justification ${ }^{38}$ as the equations in the McMillan-Mayer standard state. The advantage of the McMillan-Mayer standard state is not in its rigour but in the simpler relationship of the resulting sociation constants to integrals involving potentials of average force. The alternate theory has been derived by Hill ${ }^{38}$ starting with a " semigrand" partition function for a system at constant $P, T$, number $\left(N_{1}\right)$ of solvent molecules and solute activity. In this approach the solvent activity plays the same role that $p V$ plays in the Grand canonical partition function and $\bar{N}_{2} / N_{1}$ (which is proportional to molality) replaces $c_{2}$. The resulting equations ${ }^{38} \mathrm{can}$ be transformed to the direct analogue of eqn (12) and (14):

$$
\begin{aligned}
m_{i} & =m_{i} \gamma_{i}+\sum_{n>1} n_{i} K_{n}^{\prime} m^{n} \gamma^{n} \\
\phi & =\left[\sum_{i} m_{i} \gamma_{i}+\sum_{n>1} K_{n}^{\prime} m^{n} \gamma^{n}\right] / \sum_{i} m_{i}
\end{aligned}
$$

where $m$ is the molality, $\phi$ is the molal osmotic coefficient, and $\gamma$ is the molal activity coefficient. We shall call $K_{n}^{\prime}$ a molality scale sociation constant by analogy even though its connection with an excess concentration is not clear. Hill's derivation is for one component. We have assumed by analogy that the multi-component equations are of the same form as eqn (12) and (14). In addition, the same arguments which lead to the equations for electrolytes can be applied to the constant pressure equations.

Although it is more difficult to relate the constants in the molality equations to potentials of average force and excess concentrations, they are very much more useful for practical calculations. The following examples illustrate this utility. By analogy with eqn (20) and (21), we have

$$
\begin{gathered}
(\phi-\gamma) / m \gamma^{2}=K_{2}^{\prime}+K_{3}^{\prime} a \\
(1-\phi) / m=-B_{2}-B_{3} m-B_{4} m^{2}-\ldots
\end{gathered}
$$

where $a$ is the molal activity, $m \gamma$.

In fig. 5 the urea data are plotted according to these two equations; the difference between the two ways of representing the results is quite striking. The activity expansion requires only two terms to represent the data to $12 \mathrm{~mol} \mathrm{~kg}^{-1}$, whereas more are required in a molality expansion. This latter point is confirmed by the expressions given by Ellerton and Dunlop ${ }^{14}$ who used four coefficients to represent the data to $7 \mathrm{~mol} \mathrm{~kg}^{-1}$.

For electrolyte solutions on the molality scale, eqn (26)-(28) become

$$
\begin{gathered}
\gamma_{i} m_{i}=a_{i}=\gamma_{i}^{\circ}\left[m_{i}-\sum_{n>1} a^{n} n_{i} K_{n}^{\prime} / \gamma_{n}^{\circ}\right] \\
\gamma_{j}^{\circ}=\exp \left(-A_{\gamma}^{\prime} Z_{j}^{2} \sqrt{ } I_{\mathrm{a}}^{\prime}\right)
\end{gathered}
$$

where $A_{\gamma}^{\prime}$ is the Debye-Hückel limiting slope on the molality scale and $I_{a}^{\prime}$ is given by

$$
I_{\mathbf{a}}^{\prime}=\left(\frac{1}{2}\right)\left(\sum_{i} a_{i} Z_{i}^{2}+\sum_{n>1} a_{n} Z_{n}^{2}\right)
$$


TABLE 2.-LEAST-SQUARES FIT OF $\gamma_{ \pm}$TO EQN (34)-(36)

\begin{tabular}{|c|c|c|c|c|c|}
\hline$N^{a}$ & $\underset{/ \operatorname{mol~kg})^{-1}}{m(\max }$ & $\underset{/ \mathrm{kg} \mathrm{mol}-1}{K^{\prime}(+-)^{\circ}}$ & $\begin{array}{c}K^{\prime}(++) \\
\mid \mathrm{kg} \mathrm{mol}^{-1}\end{array}$ & $\begin{array}{l}K^{\prime}(++--) \\
/ \mathrm{kg}^{2} \mathrm{~mol}^{-2}\end{array}$ & $\stackrel{\sigma^{d}}{110^{-2}}$ \\
\hline \multicolumn{6}{|c|}{ (A) $\mathrm{CuSO}_{4}$} \\
\hline 4 & 0.01 & $199(3)^{e}$ & & & 0.06 \\
\hline 14 & 1.0 & $225(27)$ & & & 0.97 \\
\hline 11 & 0.5 & 199(11) & $0.60(26)$ & & 0.25 \\
\hline 11 & 0.5 & $195(5)$ & & $844(137)$ & 0.11 \\
\hline 11 & 0.5 & 195 & -0.047 & 903 & 0.11 \\
\hline 16 & 1.4 & 198(9) & & $676(189)$ & 0.22 \\
\hline \multicolumn{6}{|c|}{ (B) $\mathrm{KCl}$} \\
\hline 7 & 0.1 & $-1.053(46)$ & & & 0.09 \\
\hline 11 & 0.5 & $-1.055(58)$ & $-0.0137(43)$ & & 0.13 \\
\hline 11 & 0.5 & $-1.074(50)$ & & $-0.193(61)$ & 0.12 \\
\hline 11 & 0.5 & -1.085 & +0.0061 & -0.2756 & 0.12 \\
\hline 16 & 1.0 & $-1.162(40)$ & & $-0.089(20)$ & 0.23 \\
\hline
\end{tabular}

$a$ Number of data points included in the fit. ${ }^{b}$ Maximum molality included in the fit. $c$ For,the salt $\mathrm{MX}, K^{\prime}(+-)$ is $K^{\prime}(\mathrm{MX})$ and $K^{\prime}(++-)$ is $K^{\prime}\left(\mathrm{M}_{2} \mathrm{X}\right)$. ${ }^{d}$ Standard deviation of the fit. $e$ The numbers in parentheses are estimated $95 \%$ confidence limits of the last digit. Thus, $0.015(20)$ is $0.015 \pm 0.020$.

Pitzer ${ }^{39}$ has reviewed the literature data on $2: 2$ electrolytes and found that there is an extensive and particularly concordant array of experimental data for copper sulphate. Thus, this salt was chosen as an example of the use of eqn (34)-(36) for

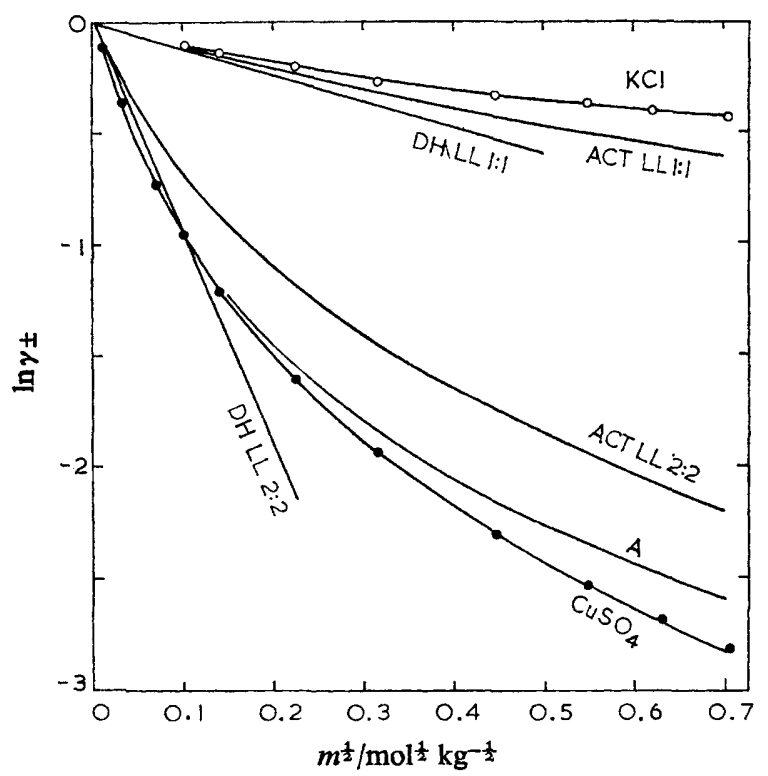

FIg. 7.-Plot of $\ln \gamma \pm$ against $m^{\frac{1}{2}}$ for $\mathrm{CuSO}_{4}$ and $\mathrm{KCl}$. The Debye-Hückel limiting law (DHLL) and activity limiting law (ACTLL) for 2:2 and 1:1 electrolytes are also given. The curve labelled $\mathrm{CuSO}_{4}$ is the best fit to the $\mathrm{CuSO}_{4}$ data $; K\left(\mathrm{CuSO}_{4}\right)=194.8 \mathrm{~kg} \mathrm{~mol}{ }^{-1}$ and $K\left(\mathrm{Cu}_{2} \mathrm{SO}_{4}^{2+}\right)=844.2 \mathrm{~kg}^{2}$ $\mathrm{mol}^{-2}$. Curve $\mathrm{A}$ is for $K\left(\mathrm{CuSO}_{4}\right)=194.8 \mathrm{~kg} \mathrm{~mol}^{-1}$ and $K\left(\mathrm{Cu}_{2} \mathrm{SO}_{4}^{2+}\right)=0$. The curve labelled $\mathrm{KCl}$ is the best fit to the $\mathrm{KCl}$ data $; K(\mathrm{KCl})=-1.074 \mathrm{~kg} \mathrm{~mol}-1$ and $K\left(\mathrm{~K}_{2} \mathrm{Cl}^{+}\right)=-0.193 \mathrm{~kg}^{2} \mathrm{~mol}^{-2}$.

The same low concentration behaviour found for $\mathrm{ZnSO}_{4}$ (fig. 6) is found for $\mathrm{CuSO}_{4}$. 
$2: 2$ electrolytes. The results of fitting these smooth data for copper sulphate are given in table 2 and fig. 7 . With one pairwise sociation constant, there are significant deviations in the fit above $0.01 \mathrm{~mol} \mathrm{~kg}{ }^{-1}$, although the data can be fit to $1 \mathrm{~mol} \mathrm{~kg}^{-1}$ with a standard deviation of only $1 \%$. Fits with two sociation constants show that a triplet term, $K^{\prime}\left(\mathrm{Cu}_{2} \mathrm{SO}_{4}^{2+}\right)$, is much more useful in fitting the data than a pair sociation constant involving the two cations, $K^{\prime}\left(\mathrm{Cu}_{2}^{4+}\right)$, especially at high concentrations. This is just what is expected because of the very strong electrostatic repulsion between the cations. With two constants, the activity coefficients can be fit to $1.4 \mathrm{~mol} \mathrm{~kg}^{-1}$ with a standard deviation of 0.0022 . These results are comparable to those obtained by Pitzer using a chemical association model with pair and triplet association and an ion-size parameter equal to the Bjerrum distance, $q$. In contrast to this, it is very difficult to fit this data with a power series in molality. Pitzer and Mayorga ${ }^{40}$ succeeded in fitting the data using a complex equation with four adjustable constants for each salt $\left(\beta_{0}, \beta_{1}, \beta_{2}\right.$, and $\left.C\right)$ together with three parameters that were adjusted to fit the data for all of the various salts $\left(b, \alpha_{1}\right.$, and $\left.\alpha_{2}\right)$. Using the resulting equation, Pitzer and Mayorga ${ }^{40}$ fitted the data for copper sulphate up to 1.4 molal with a standard deviation of 0.003 .

As an example of a 1:1 electrolyte, the smoothed activity coefficients of Hamer and $\mathrm{Wu}^{41}$ for $\mathrm{KCl}$ were fitted to the present equations. Some of the results are given in table 2 . Using only one adjustable constant gave $K^{\prime}(\mathrm{KCl})=-1.0527 \pm$ $0.045 \mathrm{~kg} \mathrm{~mol}^{-1}$ and a standard deviation of the fit of 0.0009 for 7 data points with a maximum molality of $0.1 \mathrm{~mol} \mathrm{~kg} \mathrm{~kg}^{-1}$. Similarly, using two adjustable constants gave good fits $(\sigma=0.0013)$ up to $0.5 \mathrm{~mol} \mathrm{~kg}^{-1}$. This is approximately the range of data that can be fit with a molality expansion using an extended Debye-Hückel equation. A plot of the fit is given in fig. 7 together with the Debye-Huickel limiting law and the activity limiting law. For $\mathrm{KCl}$ the activity coefficients are always above the activity limiting law so the sociation constants are always negative.

In principle, the present equations have the ability to distinguish between sociation between a cation and anion $\left[K^{\prime}\left(\mathrm{CuSO}_{4}\right)\right]$ and sociation between two cations, $\left[K^{\prime}\left(\mathrm{Cu}_{2}^{4}\right)\right]$. This is due to the difference in the charges and hence ion-atmosphere of the sociated species; one pair has a charge of zero, while the other pair has a charge of +4 . As a test of whether this distinction is feasible with real data, an attempt was made to fit the copper sulphate data with strong pairing between the cations, by choosing initial $K^{\prime}$ values which favoured $K^{\prime}\left(\mathrm{Cu}_{2}^{4+}\right)$ over $K^{\prime}\left(\mathrm{CuSO}_{4}\right)(199$ and 0.60 , respectively). When this was attempted, the least squares program was unable to find a valid solution to the equation. However, when both initial $K^{\prime}$ values were set equal to zero so as not to bias the result, the least squares program went quickly to $K^{\prime}\left(\mathrm{CuSO}_{4}\right)=199$ and $K^{\prime}\left(\mathrm{Cu}_{2}^{4}\right)=0.60$ using data with a maximum molality of $0.5 \mathrm{~mol} \mathrm{~kg} \mathrm{~kg}^{-1}$. The standard deviation of the fit was 0.0025 . Thus, at least in this case, the equations can distinguish between the two kinds of sociation. A similar test using the $\mathrm{KCl}$ data showed that the same kind of discrimination was possible.

When attempts were made to use three adjustable constants with any of the above data for electrolytes, it was found that there was very little improvement in the quality of the fit. (See table 2 for some examples.) This is because of the strong correlation between the variables. However, table 2 shows that the correlation is such that the values of $K(\mathrm{MX})$ are independent (within the $95 \%$ confidence limits) of whether $K(\mathrm{MM})$ or $K\left(\mathrm{M}_{2} \mathrm{X}\right)$ or both were also used in fitting the data. Thus, $K(\mathrm{MX})$ can be distinguished from $K(\mathrm{MM})$ and $K\left(\mathrm{M}_{2} \mathrm{X}\right)$ with good accuracy. For copper sulphate the value of $K\left(\mathrm{Cu}_{2}^{4+}\right)$ was very small, but it changed sign when a $K\left(\mathrm{Cu}_{2} \mathrm{SO}_{4}^{2+}\right)$ term was also used in the fit (see table 2). 
Similarly, the values of $K\left(\mathrm{~K}_{2}^{2+}\right)$ and $K\left(\mathrm{~K}_{2} \mathrm{Cl}^{+}\right)$changed sign as the third sociation constant was added to the fit. These results indicate that weak cation-cation sociation cannot be distinguished from three-ion sociation. In general, table 2 shows that either fit using two constants $\left[K(\mathrm{MX})+K\left(\mathrm{M}_{2}\right)\right.$ or $\left.K(\mathrm{MX})+K\left(\mathrm{M}_{2} \mathrm{X}\right)\right]$ gave about the same accuracy. It is observed, however, that the fit with only pairs tends to be slightly better with data limited to low concentrations and this changes as the concentration limit is increased. This is just the behaviour expected from the concentration dependence of these terms.

The support of this work by the National Science Foundation is gratefully acknowledged. Thanks are also due to the University of Sheffield for sabbatical leave for T. H. L. and to Swarthmore College for sabbatical leave for P. T. T.

We also wish to thank H. S. Frank, H. L. Friedman, P. Manglesdorf, K. S. Pitzer and W. Y. Wen for comments on a draft manuscript.

\section{APPENDIX}

An outline of the proof is given below. From eqn (26)-(28) it is easy to see that the sociation constants, $K_{n}$, appear in $\ln \gamma_{t}$ only in terms of order $c[\mathcal{O}(c)]$ or higher. Thus, to obtain the leading term in the activity expansion $\left[O\left(c^{\frac{1}{2}}\right)\right]$, we need not consider any terms involving $K_{n}$ or any terms of order higher than $c^{\frac{1}{2}}$. With these simplifications, eqn (26)-(28) reduce to:

$$
\begin{aligned}
\gamma_{i} & =\gamma_{i}^{\circ} \\
I_{\mathrm{a}} & =\frac{1}{2} \sum_{i} c_{i} Z_{i}^{2} \gamma_{i}^{\circ} \\
\gamma_{i}^{\circ} & =\exp \left[-A_{\gamma} Z_{i}^{2} I_{\mathrm{a}}^{\frac{1}{2}}\right] \\
& =1-A_{\gamma} Z_{i}^{2} I_{\mathrm{a}}^{\frac{1}{2}} .
\end{aligned}
$$

Therefore,

$$
\begin{aligned}
I_{\mathrm{a}} & =\frac{1}{2} \sum_{i} c_{i} Z_{i}^{2}-\frac{1}{2} \sum_{i} c_{i} Z_{i}^{4} A_{\gamma} I_{\mathrm{a}}^{\frac{1}{2}} \\
& =I+\mathcal{O}\left(c^{\frac{3}{2}}\right) .
\end{aligned}
$$

Thus, $\gamma_{i}$ reduces to $\gamma_{i}^{\circ}$ and $I_{\mathrm{a}}$ reduces to $I$. It immediately follows that $\ln \gamma_{i}=$ $-A_{\gamma} Z_{i}^{2} I^{\frac{1}{2}}$ for an ionic species and that for any binary electrolyte in $\gamma_{ \pm}=$ $-A_{\gamma}\left|Z_{+} Z_{-}\right| I^{\frac{1}{2}}$, which is the Debye-Hückel Limiting Law.

Note added in proof: The application of an activity expansion approach to plasmas has been discussed by G. P. Bartsch and W. Ebeling, Beitr. Plasmaphysik, 1971, 11, 393; F. J. Rogers and H. E. DeWitt, Phys. Rev., 1973, A8, 1061; F. J. Rogers, Phys. Rev., 1974, A10, 2441; W. Ebeling, Physica, 1974, 73, 573.

\section{GLOSSARY}

$A^{\text {ex }}=$ excess Helmholtz free energy

$a_{i}=$ activity of $i$ th species $=\gamma_{i} c_{i}$ or $\gamma m$ depending on the concentration scale

$A_{\gamma}=$ Debye-Hückel limiting slope

$B_{n}=$ virial coefficient involving the set $n$

$b_{n}=$ cluster integral of Mayer involving the set $n$

$(\mathrm{CI})_{N}=$ configuration integral for $N$ particles

$\mathrm{CPF}=$ Canonical Partition Function

$c=$ concentration

GCPF $=$ Grand Canonical Partition Function

$I=$ molar ionic strength $=\frac{1}{2} \sum_{i} c_{i} Z_{i}^{2}$ 
$I_{\mathrm{a}}=$ molar ionic strength expressed in terms of activities [eqn (28)]

$I_{a}^{\prime}=$ molal ionic strength expressed in terms of activities

$K_{n}=$ sociation constant for the molecules or ions in the set $n=b_{n} N^{(n-1)}$

$K_{n}^{\prime}=$ sociation constant on the molal scale

$\boldsymbol{k}=$ Boltzmann's constant

$N=$ Avogadro's constant

$N=$ number of molecules in the system

$\boldsymbol{n}=\mathbf{a}$ set of molecules or ions containing $n_{1}$ of species $1, n_{2}$ of species, $\ldots$ and $n_{\sigma}$ of species $\sigma$

$n_{i}=$ number of molecules or ions of species $\boldsymbol{i}$ in the set $\boldsymbol{n}$

$p=$ pressure

$p^{\text {id }}=$ pressure if the system were ideal

$\boldsymbol{R}=$ gas constant

$T=$ temperature

$U^{\mathrm{ex}}=$ excess internal energy

$V=$ volume of the system

$W=$ potential of average force

$Z_{i}=$ charge on ion $i$ or $Z_{n}=$ charge on cluster of the set $n$

$z=$ activity $=(C I)_{1} \lambda / V ; z \rightarrow N / V$ as $c \rightarrow 0$

$z^{n}=z_{1}^{n_{1}} z_{2}^{n_{2}} \ldots z^{n}$

$\gamma_{i}=a_{i} / c_{i}$ or $a_{i} / m_{i}$, depending on concentration scale

$\gamma_{i}^{\circ}=$ corrections for screening of species $i$ by the ion atmosphere

$\rho=N / V=$ particle number density

$\mu=$ chemical potential

$\lambda=$ absolute activity $=\exp (\mu / k T)$

$\phi=$ osmotic coefficient for a solution or compressibility factor for a gas

${ }^{1}$ J. M. Prausnitz, Molecular Thermodynamics of Fluid-phase Equilibria (Prentice-Hall, 1969).

2 Some classic examples of the use of this approach are: $(a)$ R. M. Garrels and M. E. Thompson, Amer. J. Sci., 1962, 260, 57 and (b) R. M. Pytkowicz and D. R. Kester, Amer. J. Sci., 1969, 267, 217.

${ }^{3}$ For instance, R. A. Robinson and R. H. Wood, J. Solution Chem., 1972, 1, 481.

A recent example of a treatment including both kinds of interactions is: K. S. Pitzer and L. F. Silvester, J. Solution Chem., 1976, 5, 269.

${ }^{4}$ H. L. Friedman and W. D. T. Dale, Modern Theoretical Chemistry, Statistical Mechanics, pt. A, ed. B. J. Berne (Plenum Press, N.Y., 1977), vol. IV, chap. 3.

5 T. L. Hill, An Introduction to Statistical Thermodynamics (Addison-Wesley, N.Y., 1960), chap. 15.

6 W. G. McMillan and J. E. Mayer, J. Chem. Phys., 1945, 13, 276.

7 J. E. Mayer and M. G. Mayer, Statistical Mechanics (Wiley, New York, 1940), chap. 13.

8 J. E. Mayer, Equilibrium Statistical Mechanics (Pergamon Press, Oxford, 1968), chap. 4.

9 E. A. Guggenheim, Trans. Faraday Soc., 1960, 56, 1159.

${ }^{10}$ T. L. Hill, Statistical Mechanics (McGraw-Hill, New York, 1956), chap. 5.

11 J. Hilsenrath, H. J. Hoge, C. W. Beckett, J. F. Masi, W. S. Benedict, R. L. Nuttall, L. Fano, Y. S. Touloukian and H. W. Woolley, Tables of Thermodynamic and Transport Properties (Pergamon Press, New York, 1960).

12 D. R. Douslin, R. H. Harrison, R. T. Moore and J. P. McCullough, J. Chem. Eng. Data, $1964,9,358$.

${ }^{13}$ F. H. Verhoek and F. Daniels, J. Amer. Chem. Soc., 1931, 53, 1250.

${ }_{14}$ H. D. Ellerton and P. J. Dunlop, J. Phys. Chem., 1966, 70, 1831.

${ }_{15}$ R. H. Stokes, Austral. J. Chem., 1967, 20, 2087.

16 H. L. Friedman, J. Solution Chem., 1972, 1, 387.

${ }^{17}$ E. P. Perman and W. D. Urry, Proc. Roy. Soc. A, 1930, 126, 44.

${ }^{18} \mathrm{H}$. L. Friedman and C. V. Krishnan, in Water: A Comprehensive Treatise, ed. F. Franks (Plenum Press, New York, 1973), vol. III, chap. 1, p. 114.

${ }^{19}$ (a) I. M. Klotz and J. S. Franzen, J. Amer. Chem. Soc., 1962, 84, 3461 ; (b) E. G. Finer, F. Franks and M. J. Tait, J. Amer. Chem. Soc., 1972, 94, 4424.

${ }^{20}$ H. C. Anderson, J. Chem. Phys., 1973, 59, 4714. 
21 J. A. Schellman, Compt. rend. Trav. Lab. Carlsberg Ser. Chim., 1955, 29, 223.

22 P. O. P. Ts'o, I. S. Melvin and A. C. Olson, J. Amer. Chem. Soc., 1963, 85, 1289.

23 H. L. Friedman, J. Chem. Phys., 1960, 32, 1351.

${ }^{24}$ H. L. Friedman, Mol. Phys., 1959, 2, 190, 436.

${ }^{25}$ H. L. Friedman, Ionic Solution Theory (Interscience, New York, 1962).

26 (a) A. A. Vedenov, J. Exptl. Theor. Phys. (U.S.S.R.), 1959, 36, 942; (b) A. A. Vedenov and A. I. Larkin, J. Exptl. Theor. Phys. (U.S.S.R.), 1959, 36, 1133.

${ }^{27}$ R. W. Jones and F. Mohling, J. Phys. Chem., 1971, 75, 3790.

${ }^{28}$ Some problems with ref. (27) have been pointed out by H. L. Friedman, J. Phys. Chem., $1972,76,1229$, but they do not appear to invalidate the limiting law.

29 R. A. Robinson, R. H. Wood and P. J. Reilly, J. Chem. Thermodynamics, 1971, 3, 461.

${ }^{30}$ (a) J. C. Rasaiah, The Activity and Osmotic Coefficients of Zinc Sulfate in Water and Heavy Water at $25^{\circ}$ C, Ph.D. Thesis (University of Pittsburgh, 1965); (b) J. C. Rasaiah, J. Solution Chem., 1973, 2, 301.

31 H. S. Frank, in discussion of paper of J. C. Rasaiah, J. Solution Chem., 1973, $2,337$.

32 P. G. M. Brown and J. E. Prue, Proc. Roy. Soc. A, 1955, 232, 320.

${ }^{33}$ T. H. Lilley and C. C. Briggs, Proc. Roy. Soc. A, 1976, 349, 355.

${ }^{34}$ S. R. Gupta, G. J. Hills and D. J. G. Ives, Trans. Faraday Soc., 1963, 59, 1874.

35 The most recent higher order limiting law would not be built into the equations. See H. L. Friedman, J. Phys. Chem., 1974, 78, 1927.

${ }^{36}$ P. S. Ramanathan and H. L. Friedman, J. Chem. Phys., 1971, 54, 1086.

37 A. S. Levine and R. H. Wood, J. Phys. Chem., 1973, 77, 2390.

${ }^{38}$ Ref. (5), p. 362 and following; especially eqn (19-81) and (19-84).

${ }^{39}$ K. S. Pitzer, J.C.S. Faraday II, 1972, 68, 101.

${ }^{40}$ K. S. Pitzer and G. Mayorga, J. Solution Chem., 1974, 3, 539.

${ }^{41}$ W. J. Hamer and Y.-C. Wu, J. Phys. Chem. Ref. Data, 1972, 1, 1047.

(PAPER 7/1632) 